\title{
Establishing an Improved Kane Dynamic Model for the 7-DOF Reconfigurable Modular Robot
}

\author{
Xiao Li, ${ }^{1}$ Hanxu Sun, ${ }^{2}$ Linjing Liao, ${ }^{2}$ and Jingzhou Song ${ }^{2}$ \\ ${ }^{1}$ School of Mechanical Engineering and Automation, Beihang University, Beijing 100191, China \\ ${ }^{2}$ School of Automation, Beijing University of Posts and Telecommunication, Beijing, China \\ Correspondence should be addressed to Xiao Li; lxwishyou@163.com
}

Received 30 September 2016; Revised 26 November 2016; Accepted 6 December 2016; Published 12 January 2017

Academic Editor: Yan-Wu Wang

Copyright (c) 2017 Xiao Li et al. This is an open access article distributed under the Creative Commons Attribution License, which permits unrestricted use, distribution, and reproduction in any medium, provided the original work is properly cited.

\begin{abstract}
We propose an improved Kane dynamic model theory for the 7-DOF modular robot in this paper, and the model precision is improved by the improved function $T^{\prime}{ }_{i}(t)$. We designed three types of progressive modular joints for reconfigurable modular robot that can be used in industrial robot, space robot, and special robot. The Kane dynamic model and the solid dynamic model are established, respectively, for the 7-DOF modular robot. After that, the experimental results are obtained from the simulation experiment of typical task in the established dynamic models. By the analysis model of error, the equation of the improved torque $T^{\prime}{ }_{i}(t)$ is derived and proposed. And the improved Kane dynamic model is established for the modular robot that used $T^{\prime}{ }_{i}(t)$. Based on the experimental data, the undetermined coefficient matrix is five-order linear that was proved in 7-DOF modular robot. And the explicit formulation is solved of the Kane dynamic model and can be used in control system.
\end{abstract}

\section{Introduction}

Reconfigurable modular robot system (RMRS) consists of a series of different functions, characteristics, and assembly function of the standard size of joint or link modules, the way to the building blocks of robotic systems that have been assembled [1]. This combination is not a simple mechanical restructuring, including control system, electronic hardware, control algorithm, and software, such as restructuring. Because the modular joint itself is a set of drive, driving, control, and communication as a whole unit [2], each modular joint is an independent movement unit and structural unit, and each module has automatically servo control. A member of joint can complete a variety of configurations and can adapt to a variety of requirements and the environment. Even if the individual joint module fails, it can also be immediately replaced with other modules. So the modular robot has the characteristics of good reconstruction, convenient assembly, good flexibility, functional diversity, strong adaptability, and being easy to maintain. And modular robot has great prospects for development and potential applications in the agile manufacturing system, laboratory research, military, aerospace, nuclear industry, extreme environment, and industrial areas [3].

In the whole robot system, modular joint is a critical part of reconfigurable modular robot. It is the foundation of the complex tasks such as the robot path planning. The stand or fall of its performance directly affects the performance, the holistic movement of a robot. So the design of joint structure and motion control system are important research tasks of the reconfigurable modular robot. At present, our research team has developed a set of modular joints that have high performance. The shapes of joints size are $146 \times 146 \times 293 \mathrm{~mm}$, $110 \times 110 \times 221 \mathrm{~mm}, 90 \times 90 \times 181 \mathrm{~mm}$, and maximum output torque of $580 \mathrm{~nm}$; the highest speed can be designed at $80 \mathrm{RPM}$.

This kind of joints can be flexible refactoring, forming 5-DOF, 6-DOF, or 7-DOF industrial robots, and meet the demand of industrial production and manufacturing [4]. 


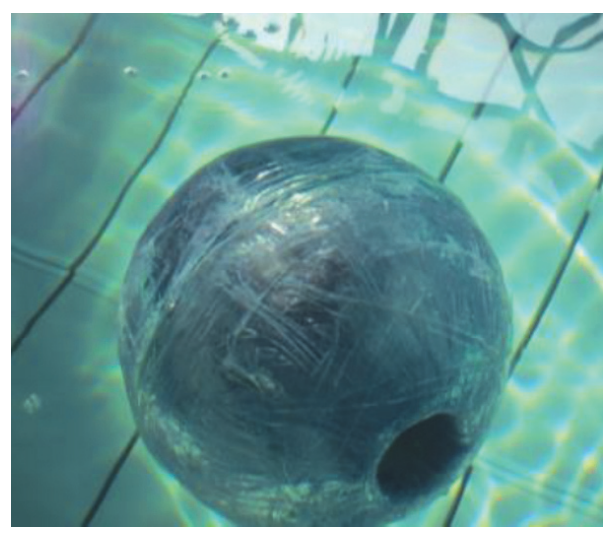

FIGURE 1: The spherical underwater robot.

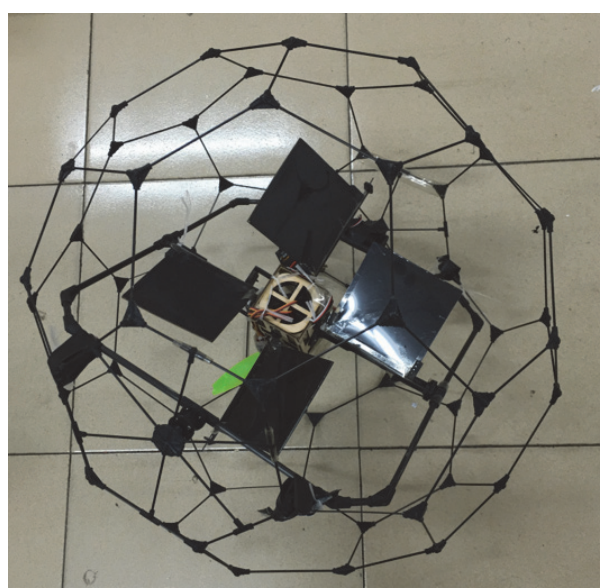

FIgURE 2: The flight spherical robot.

Modular robot can also be applied to spherical underwater robot $[5,6]$, as spherical underwater robot drive unit and heavy pendulum control unit. Spherical underwater robot is shown in Figure 1. Light joints can also be used for the flight spherical robot pose [7] and become the center of gravity control unit. Flight spherical robot is shown in Figure 2. Modular joint can also be used for space robot reconfigurable, research for the space robot control technology, and experimental performance [8].

In the modular robot control system, kinematics and dynamics model is crucial and important. Kinematics description methods are common D-H parameters method, the improved method of D-H parameters, exponential product formula method (POE), and so forth $[9,10]$. Modular robot dynamics modeling methods are the Newton-Euler method [11], Lagrange method [12], Kane method [13, 14], the principle of virtual work method [15], spinor even method [16], and so forth. The characteristics of the NewtonEuler method and comprehensive calculation are detailed, when multiple degrees of freedom model is too complicated. The Lagrange method is to avoid the calculation of linear acceleration and angular acceleration, but when the freedom of the robot is high the calculation is larger. Therefore the dynamic model is often simplified or we ignore inertia effect in the actual application [17-19]. The characteristic of the Kane method is that the sum of generalized force and generalized inertia force is zero. And The Kane method is to avoid the calculation of derivative, and it has high calculation efficiency and facilitates computer control [2022]. At the same time, the Kane method is also applied to dynamics modeling and high precision control of parallel manipulator [23-25] and has achieved good results. In view of this, we used the modeling of Kane method in this paper.

In the general dynamic model, the mathematical model is less accurate than the solid model, but can be used directly in the control system. The solid model accuracy is high but cannot be used for direct control of the control system.

In this paper, we propose an improved Kane model theory for the 7-DOF modular robot, and the dynamic model precision is improved by the modified function $T^{\prime}{ }_{i}(t)$. 


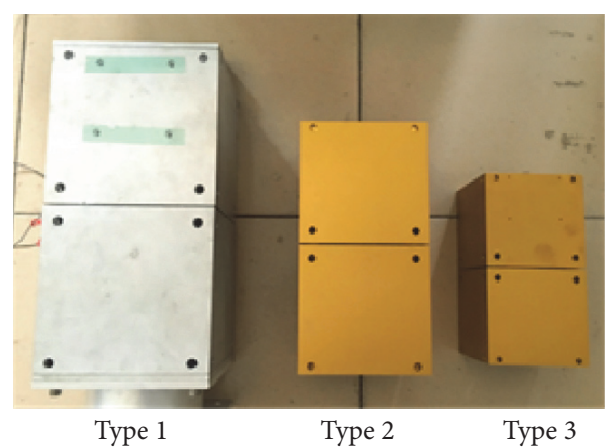

Figure 3: The type of modular joint.

Based on the analysis of the error between dynamic and solid models, the dynamic correction model with modified function $\left(T^{\prime}{ }_{i}(t)\right)$ was established. The Kane model is used to obtain the result of the mathematical model. And the Adams model is the result of the implementation of the solid model. Comparing and analyzing the results of the two models, we can get the undetermined coefficient matrix $C_{i j}$ of the modified function. After that, it can use the correction function to improve the accuracy of the dynamic model. The experimental results show that the modified function can effectively improve the accuracy of the dynamic model. And the modification function $T^{\prime}(t)$ is five-order linear in the 7DOF modular robot that was designed.

\section{The Modular Robot Design Parameter and Kinematic Model}

Our research team design a 7-DOF modular reconfigurable robot and the modular joint is shown in Figure 3. The basic module consists of three types of joints: Type 1, Type 2, and Type 3. These joints can also be designed in accordance with the requirements into larger or smaller type. The appearance size of Type 1 is $146 \times 146 \times 293 \mathrm{~mm}$, the appearance size of Type 2 is $110 \times 110 \times 221 \mathrm{~mm}$, and the appearance size of Type 3 is $90 \times 90 \times 181 \mathrm{~mm}$. The design configuration of 7-DOF modular reconfigurable robot is shown in Figure 4. Among them Joint 1 and Joint 2 are Type 1, Joint 3 and Joint are Type 2, and Joint 5, Joint 6, and Joint 7 are Type 3 . The flange length, in turn, is $54 \mathrm{~mm}, 108 \mathrm{~mm}, 60 \mathrm{~mm}, 60 \mathrm{~mm}, 60 \mathrm{~mm}$, and $60 \mathrm{~mm}$ from Joint 1 to Joint 7.

Joint design parameters are shown in Table 1 . We can see the joints support flexible way of refactoring.

By the robot configuration, design parameters, and size parameters, the D-H parameters of 7-DOF modular robot can be determined, as shown in Table 2 .

By the D-H parameters, the kinematic model is established for 7-DOF modular robot. It is expressed as

$$
{ }_{i}^{i-1} T=\left[\begin{array}{cccc}
c \theta_{i} & -s \theta_{i} & 0 & a_{i-1} \\
s \theta_{i} c \alpha_{i-1} & c \theta_{i} c \alpha_{i-1} & -s \alpha_{i-1} & -d_{i} s \alpha_{i-1} \\
s \theta_{i} s \alpha_{i-1} & c \theta_{i} s \alpha_{i-1} & c \alpha_{i-1} & d_{i} c \alpha_{i-1} \\
0 & 0 & 0 & 1
\end{array}\right], \quad, \quad i=1 \sim 7 .
$$

The transformation matrix of each joint is expressed as

$$
\begin{aligned}
A_{1} & =\left[\begin{array}{cccc}
c \theta_{1} & -s \theta_{1} & 0 & 0 \\
s \theta_{1} & c \theta_{1} & 0 & 0 \\
0 & 0 & 1 & 420 \\
0 & 0 & 0 & 1
\end{array}\right], \\
A_{2} & =\left[\begin{array}{cccc}
c \theta_{2} & -s \theta_{2} & 0 & 0 \\
0 & 0 & -1 & -147 \\
s \theta_{2} & c \theta_{2} & 0 & 0 \\
0 & 0 & 0 & 1
\end{array}\right], \\
A_{7} & =\left[\begin{array}{cccc}
c \theta_{3} & -s \theta_{3} & 0 & 0 \\
0 & 0 & 1 & 535 \\
-s \theta_{3} & -c \theta_{3} & 0 & 0 \\
0 & 0 & 0 & 1
\end{array}\right], \\
A_{6} & {\left[\begin{array}{cccc}
c \theta_{7} & -s \theta_{7} & 0 & 0 \\
0 & 0 & 1 & 91 \\
-s \theta_{7} & -c \theta_{7} & 0 & 0 \\
0 & 0 & 0 & 1
\end{array}\right] } \\
A_{4}= & {\left[\begin{array}{cccc}
c \theta_{4} & -s \theta_{4} & 0 & 0 \\
0 & 0 & 1 & 0 \\
-s \theta_{6} & -c \theta_{6} & 0 & 0 \\
0 & -c \theta_{4} & 0 & 0 \\
0 & 0 & 0 & 1
\end{array}\right], } \\
A_{5}= & {\left[\begin{array}{cccc}
c \theta_{5} & -s \theta_{5} & 0 & 170 \\
-s \theta_{5} & -c \theta_{5} & 0 & 0 \\
0 & 0 & -1 & 0 \\
0 & 0 & 0 & 1
\end{array}\right], }
\end{aligned}
$$

Therefore, the kinematic model is expressed as Formula (3) for the 7-DOF modular robot.

$$
T=A_{1} \cdot A_{2} \cdot A_{3} \cdot A_{4} \cdot A_{5} \cdot A_{6} \cdot A_{7} .
$$

\section{Establish Dynamical Model and Simulation}

First, the modeling parameters are determined by the measurement and calculation. The mass of joints is expressed as follows:

$$
\begin{aligned}
& {\left[\begin{array}{lllllll}
m_{1} & m_{2} & m_{3} & m_{4} & m_{5} & m_{6} & m_{7}
\end{array}\right]^{T}} \\
& \quad=\left[\begin{array}{lllllll}
13.6 & 13.6 & 9.79 & 5.95 & 4.64 & 3.33 & 1.52
\end{array}\right]^{T} .
\end{aligned}
$$


TABLE 1: Joint design parameters.

\begin{tabular}{lcccc}
\hline Designation & Unit & Type 1 & Type 2 & Type 3 \\
\hline Rated torque & $\mathrm{Nm}$ & 500 & 200 & 110 \\
Peak torque & $\mathrm{Nm}$ & 1400 & 800 & 400 \\
Rotation angle & $\circ$ & $>360$ & $>360$ & 7360 \\
Weight & $\mathrm{kg}$ & 13 & $\leq 0.002$ & 5.5 \\
Repeated accuracy & $\circ$ & $\leq 0.002$ & 75 & $\leq 0.002$ \\
Maximum angular velocity & $\mathrm{rpm}$ & 35 & $160 / 120$ & 80 \\
Reduction ratio & 1 & $160 / 120$ & $24 / 48$ & $160 / 120$ \\
Nominal voltage & VDC & $24 / 48$ & RS232; CAN-Bus; USB & RS232; CAN-Bus; USB \\
Electrical interface & & RS232; CAN-Bus; USB & &
\end{tabular}

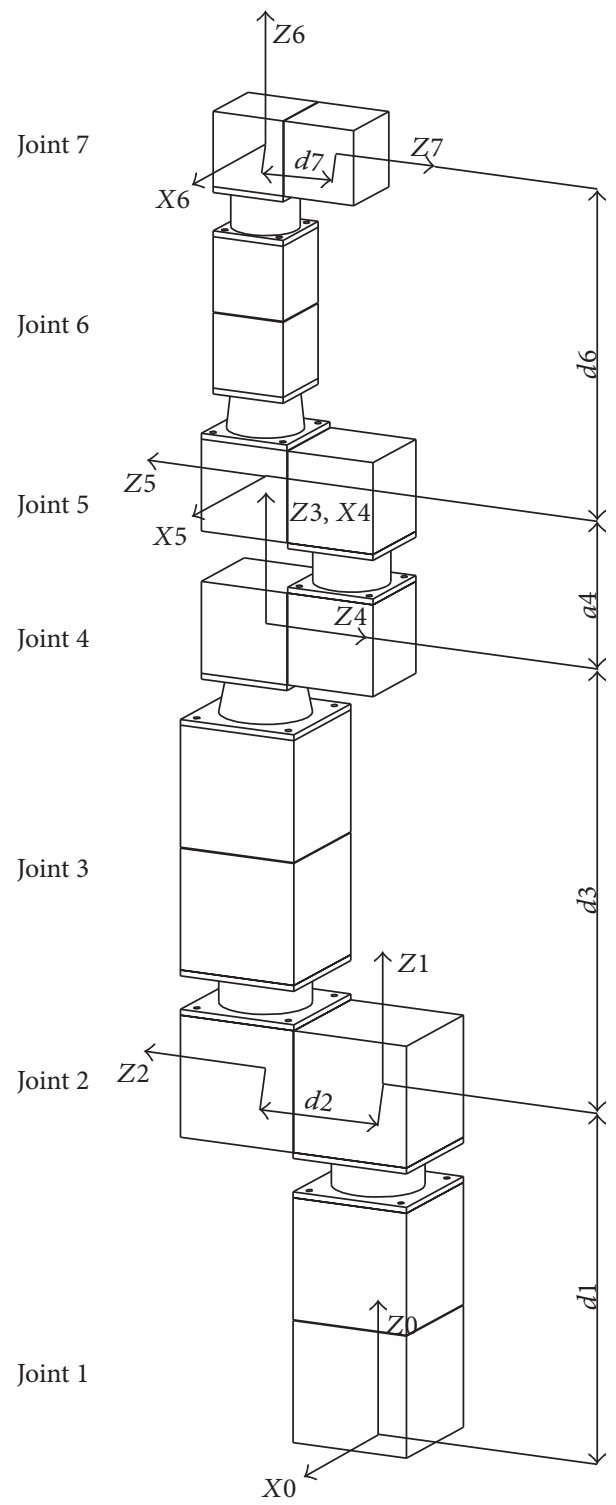

FIGURE 4: The design configuration of 7-DOF modular reconfigurable robot. 
TABLE 2: The D-H parameters of 7-DOF modular robot.

\begin{tabular}{lcccc}
\hline Link $i$ & $\alpha_{i-1}$ & $a_{i-1} / \mathrm{mm}$ & $d_{i} / \mathrm{mm}$ & 420 \\
\hline 1 & 0 & 0 & 147 & 0 \\
2 & $\mathrm{pi} / 2$ & 0 & 535 & 0 \\
3 & $0-\mathrm{pi} / 2$ & 0 & 0 & 0 \\
4 & $0-\mathrm{pi} / 2$ & 0 & 0 & $0-\mathrm{pi} / 2$ \\
5 & $\mathrm{pi}$ & 170 & 401 & 0 \\
6 & $0-\mathrm{pi} / 2$ & 0 & 91 & 0 \\
7 & $0-\mathrm{pi} / 2$ & 0 & & 0
\end{tabular}

The location of mass center is expressed as Formula (5) by automatic measurement in Proe solid model.

$$
\begin{aligned}
{\left[\begin{array}{lllllll}
r_{1} & r_{2} & r_{3} & r_{4} & r_{5} & r_{6} & r_{7}
\end{array}\right] } \\
\quad=\left[\begin{array}{ccccccc}
0 & 0 & 0 & 85 & 0 & 0 & 0 \\
0 & 100 & 0 & 0 & 58.43 & 0 & 0 \\
-100 & 0 & -129.533 & 111 & 0 & -75 & 0
\end{array}\right] .
\end{aligned}
$$
follows:

The rotation matrix of transfer matrix is expressed as

$$
\begin{aligned}
& { }_{0}^{1} R=\left[\begin{array}{ccc}
c \theta_{1} & -s \theta_{1} & 0 \\
s \theta_{1} & c \theta_{1} & 0 \\
0 & 0 & 1
\end{array}\right], \\
& { }_{1}^{2} R=\left[\begin{array}{ccc}
c \theta_{2} & -s \theta_{2} & 0 \\
0 & 0 & -1 \\
s \theta_{2} & c \theta_{2} & 0
\end{array}\right] \text {, } \\
& { }_{2}^{3} R=\left[\begin{array}{ccc}
c \theta_{3} & -s \theta_{3} & 0 \\
0 & 0 & 1 \\
-s \theta_{3} & -c \theta_{3} & 0
\end{array}\right], \\
& { }_{3}^{4} R=\left[\begin{array}{ccc}
c \theta_{4} & -s \theta_{4} & 0 \\
0 & 0 & 1 \\
-s \theta_{4} & -c \theta_{4} & 0
\end{array}\right] \text {, } \\
& { }_{4}^{5} R=\left[\begin{array}{ccc}
c \theta_{5} & -s \theta_{5} & 0 \\
-s \theta_{5} & -c \theta_{5} & 0 \\
0 & 0 & -1
\end{array}\right] \text {, } \\
& { }_{5}^{6} R=\left[\begin{array}{ccc}
c \theta_{6} & -s \theta_{6} & 0 \\
0 & 0 & 1 \\
-s \theta_{6} & -c \theta_{6} & 0
\end{array}\right], \\
& { }_{6}^{7} R=\left[\begin{array}{ccc}
c \theta_{7} & -s \theta_{7} & 0 \\
0 & 0 & 1 \\
-s \theta_{7} & -c \theta_{7} & 0
\end{array}\right] .
\end{aligned}
$$

Inertia tensor is expressed as the following Formulas from (7a) to $(7 \mathrm{~g})$ about each joint in their joint coordinate system. And the units are $\mathrm{kg} \cdot \mathrm{mm}^{2}$.

$$
\begin{aligned}
& I_{1}=\left[\begin{array}{ccc}
I_{x x 1} & I_{x y 1} & I_{x z 1} \\
I_{y x 1} & I_{y y 1} & I_{y z 1} \\
I_{z x 1} & I_{z y 1} & I_{z z 1}
\end{array}\right]=\left[\begin{array}{ccc}
313.65 & 0 & 0 \\
0 & 313.65 & 0 \\
0 & 0 & 48.61
\end{array}\right] \text {, } \\
& I_{2}=\left[\begin{array}{ccc}
I_{x x 2} & I_{x y 2} & I_{x z 2} \\
I_{y x 2} & I_{y y 2} & I_{y z 2} \\
I_{z x 2} & I_{z y 2} & I_{z z 2}
\end{array}\right]=\left[\begin{array}{ccc}
313.65 & 0 & 0 \\
0 & 48.61 & 0 \\
0 & 0 & 313.65
\end{array}\right] \text {, } \\
& I_{3}=\left[\begin{array}{ccc}
I_{x x 3} & I_{x y 3} & I_{x z 3} \\
I_{y x 3} & I_{y y 3} & I_{y z 3} \\
I_{z x 3} & I_{z y 3} & I_{z z 3}
\end{array}\right]=\left[\begin{array}{ccc}
263.69 & 0 & 0 \\
0 & 263.69 & 0 \\
0 & 0 & 30.38
\end{array}\right] \text {, } \\
& I_{4}=\left[\begin{array}{ccc}
I_{x x 4} & I_{x y 4} & I_{x z 4} \\
I_{y x 4} & I_{y y 4} & I_{y z 4} \\
I_{z x 4} & I_{z y 4} & I_{z z 4}
\end{array}\right] \\
& =\left[\begin{array}{ccc}
854.27 & 0 & -56.14 \\
0 & 168.32 & 0 \\
-56.14 & 0 & 95.01
\end{array}\right] \text {, } \\
& I_{5}=\left[\begin{array}{ccc}
I_{x x 5} & I_{x y 5} & I_{x z 5} \\
I_{y x 5} & I_{y y 5} & I_{y z 5} \\
I_{z x 5} & I_{z y 5} & I_{z z 5}
\end{array}\right]=\left[\begin{array}{ccc}
49.37 & 0 & 0 \\
0 & 8.34 & 0 \\
0 & 0 & 49.37
\end{array}\right] \text {, } \\
& I_{6}=\left[\begin{array}{ccc}
I_{x x 6} & I_{x y 6} & I_{x z 6} \\
I_{y x 6} & I_{y y 6} & I_{y z 6} \\
I_{z x 6} & I_{z y 6} & I_{z z 6}
\end{array}\right]=\left[\begin{array}{ccc}
40.32 & 0 & 0 \\
0 & 40.32 & 0 \\
0 & 0 & 4.57
\end{array}\right] \text {, } \\
& I_{7}=\left[\begin{array}{lll}
I_{x x 7} & I_{x y 7} & I_{x z 7} \\
I_{y x 7} & I_{y y 7} & I_{y z 7} \\
I_{z x 7} & I_{z y 7} & I_{z z 7}
\end{array}\right]=\left[\begin{array}{ccc}
2.06 & 0 & 0 \\
0 & 2.05 & 0 \\
0 & 0 & 2.05
\end{array}\right] \text {. }
\end{aligned}
$$


According to the configuration Figure 4 and size parameters, the equation of generalized displacement is expressed as the following Formula (8). And the units are $\mathrm{mm}$.

$$
\begin{aligned}
\widetilde{P}_{i}= & {\left[\begin{array}{lllllll}
\widetilde{P_{1}} & \widetilde{P_{2}} & \widetilde{P_{3}} & \widetilde{P_{4}} & \widetilde{P_{5}} & \widetilde{P_{6}} & \widetilde{P_{7}}
\end{array}\right] } \\
= & {\left[\begin{array}{ccccccc}
0 & 0 & 0 & 0 & 170 & 0 & 0 \\
0 & 0 & 0 & 0 & 0 & 0 & 0 \\
420 & 147 & 535 & 0 & 0 & 401 & 91
\end{array}\right] . }
\end{aligned}
$$

The generalized velocity and the generalized acceleration are derived by the Kane dynamic method, from Joint 1 to Joint 7. The main calculation formulas are expressed as the following Formula (9).

The generalized angular velocity $\widetilde{\omega}_{i}$ is expressed as Formula (9). The generalized angular velocity of partial derivative is expressed as Formula (10). The generalized velocity is expressed as Formula (11). The barycenter of generalized velocity is expressed as Formula (12). And the partial derivative of (12) is expressed as Formula (13). The generalized angular acceleration is expressed as Formula (14). The generalized acceleration is expressed as Formula (15). The barycenter of generalized acceleration is expressed as Formula (16).

$$
\begin{aligned}
\widetilde{\omega}_{i} & ={ }_{i-1}^{i} R\left(\widetilde{\omega}_{i-1}+\dot{\theta}_{i} e_{0}\right), \\
\widetilde{\omega}_{i, \dot{\theta}_{j}} & =\frac{d \widetilde{\omega}_{i}}{d \dot{\theta}_{j}}, \\
\widetilde{v}_{i} & =\widetilde{\omega}_{i} \times \widetilde{P}_{i}+{ }_{i-1}^{i} R \widetilde{v}_{i-1}, \\
\widetilde{v}_{C i} & =\widetilde{v}_{i}+\widetilde{\omega}_{i} \times R_{i}, \\
\widetilde{v}_{c i, \dot{\theta}_{j}} & =\frac{d \widetilde{v}_{C i}}{d \dot{\theta}_{j}}, \\
\widetilde{\dot{\omega}}_{i} & ={ }_{i-1}^{i} R\left(\widetilde{\dot{\omega}}_{i-1}+\omega_{i-1} \times \dot{\theta}_{i} e_{0}+\ddot{\theta}_{i} e_{0}\right), \\
\widetilde{\dot{v}}_{i} & ={ }_{i-1}^{i} R \widetilde{\dot{v}}_{i-1}+\widetilde{\dot{\omega}}_{i} \times \widetilde{P}_{i}+\widetilde{\omega}_{i} \times\left(\widetilde{\omega}_{i} \times \widetilde{P}_{i}\right), \\
\widetilde{\dot{v}}_{c i} & =\widetilde{\dot{v}}_{i}+\widetilde{\dot{\omega}}_{i} \times \widetilde{R}_{i}+\widetilde{\omega}_{i} \times\left(\widetilde{\omega}_{i} \times \widetilde{P}_{i}\right) .
\end{aligned}
$$

The initial conditions of the 7-DOF modular robot are expressed as follows:

$$
\begin{aligned}
& \omega_{0}=0, \\
& \dot{\omega}_{0}=0, \\
& v_{0}=0, \\
& \dot{v_{0}}=\left[\begin{array}{lll}
0 & 0 & g
\end{array}\right]^{T} .
\end{aligned}
$$

The main dynamic equation is expressed as the following Formula (19). And (18) is the calculation parameter of Formula (19).

$$
\begin{aligned}
& \widetilde{N}_{n}=\left(I_{n} \tilde{\dot{\omega}}_{n}+\widetilde{\omega_{n}} \times\left(I_{n} \cdot \widetilde{\omega}_{n}\right)\right)^{T}, \\
& \tau_{\theta_{j}}=\sum_{i=j}^{n} m_{i} \widetilde{\dot{v}}_{c i}^{T} \cdot v_{c i, \dot{\theta} j}+\sum_{i=j}^{n} \widetilde{N}_{i} \widetilde{\omega}_{i, \dot{\theta} j} .
\end{aligned}
$$

In order to simplify the calculation model, the dynamic model does not impose additional external force load at first. According to Formula (18) and Formula (19), the torque calculation results are calculated by putting the known parameter into the equations.

We can get that the final torque equations for the 7-DOF modular robot which are expressed as follows:

$$
\begin{aligned}
& \tau_{1}=m_{1} \tilde{\dot{v}}_{c i}^{T} \cdot v_{c 1, \dot{\theta} 1}+m_{2} \tilde{\dot{v}}_{c 2}^{T} \cdot v_{c 2, \dot{\theta} 1}+m_{3} \tilde{\dot{v}}_{c 3}^{T} \cdot v_{c 3, \dot{\theta} 1} \\
& +m_{4} \tilde{\dot{v}}_{c 4}{ }^{T} \cdot v_{c 4, \dot{\theta} 1}+m_{5} \tilde{\dot{v}}_{c 5}^{T} \cdot v_{c 5, \dot{\theta} 1}+m_{6} \tilde{\dot{v}}_{c 6}{ }^{T} \\
& \cdot v_{c 6, \dot{\theta} 1}+m_{7} \tilde{\dot{v}}_{c 7}^{T} \cdot v_{c 7, \dot{\theta} 1}+\widetilde{N}_{1} \widetilde{\omega}_{1, \dot{\theta} 1}+\widetilde{N}_{2} \widetilde{\omega}_{2, \dot{\theta} 1} \\
& +\widetilde{N}_{3} \widetilde{\omega}_{3, \dot{\theta} 1}+\widetilde{N}_{4} \widetilde{\omega}_{4, \dot{\theta} 1}+\widetilde{N}_{5} \widetilde{\omega}_{5, \dot{\theta} 1}+\widetilde{N}_{6} \widetilde{\omega}_{6, \dot{\theta} 1} \\
& +\widetilde{N}_{7} \widetilde{\omega}_{7, \dot{\theta} 1} \\
& \tau_{2}=m_{2} \tilde{\dot{v}}_{c 2}^{T} \cdot v_{c 2, \dot{\theta} 2}+m_{3} \tilde{\dot{v}}_{c 3}^{T} \cdot v_{c 3, \dot{\theta} 2}+m_{4} \tilde{\dot{v}}_{c 4}^{T} \cdot v_{c 4, \dot{\theta} 2} \\
& +m_{5} \tilde{\dot{v}}_{c 5}{ }^{T} \cdot v_{c 5, \dot{\theta} 2}+m_{6} \tilde{\dot{v}}_{c 6}{ }^{T} \cdot v_{c 6, \dot{\theta} 2}+m_{7} \tilde{\dot{v}}_{c 7}{ }^{T} \\
& \cdot v_{c 7, \dot{\theta} 2}+\widetilde{N}_{2} \widetilde{\omega}_{2, \dot{\theta} 2}+\widetilde{N}_{3} \widetilde{\omega}_{3, \dot{\theta} 2}+\widetilde{N}_{4} \widetilde{\omega}_{4, \dot{\theta} 2} \\
& +\widetilde{N}_{5} \widetilde{\omega}_{5, \dot{\theta} 2}+\widetilde{N}_{6} \widetilde{\omega}_{6, \dot{\theta} 2}+\widetilde{N}_{7} \widetilde{\omega}_{7, \dot{\theta} 2} \\
& \tau_{3}=m_{3} \widetilde{\dot{v}}_{c 3}{ }^{T} \cdot v_{c 3, \dot{\theta} 3}+m_{4} \widetilde{\dot{v}}_{c 4}{ }^{T} \cdot v_{c 4, \dot{\theta} 3}+m_{5} \widetilde{\dot{v}}_{c 5}{ }^{T} \cdot v_{c 5, \dot{\theta} 3} \\
& +m_{6} \tilde{\dot{v}}_{c 6}^{T} \cdot v_{c 6, \dot{\theta} 3}+m_{7} \tilde{\dot{v}}_{c 7}^{T} \cdot v_{c 7, \dot{\theta} 3}+\widetilde{N}_{3} \widetilde{\omega}_{3, \dot{\theta} 3} \\
& +\widetilde{N}_{4} \widetilde{\omega}_{4, \dot{\theta} 3}+\widetilde{N}_{5} \widetilde{\omega}_{5, \dot{\theta} 3}+\widetilde{N}_{6} \widetilde{\omega}_{6, \dot{\theta} 3}+\widetilde{N}_{7} \widetilde{\omega}_{7, \dot{\theta} 3}, \\
& \tau_{4}=m_{4} \tilde{\dot{v}}_{c 4}^{T} \cdot v_{c 4, \dot{\theta} 4}+m_{5} \tilde{\dot{v}}_{c 5}^{T} \cdot v_{c 5, \dot{\theta} 4}+m_{6} \tilde{\dot{v}}_{c 6}^{T} \cdot v_{c 6, \dot{\theta} 4} \\
& +m_{7} \widetilde{\dot{v}}_{c 7}^{T} \cdot v_{c 7, \dot{\theta} 4}+\widetilde{N}_{4} \widetilde{\omega}_{4, \dot{\theta} 4}+\widetilde{N}_{5} \widetilde{\omega}_{5, \dot{\theta} 4} \\
& +\widetilde{N}_{6} \widetilde{\omega}_{6, \dot{\theta} 4}+\widetilde{N}_{7} \widetilde{\omega}_{7, \dot{\theta} 4},
\end{aligned}
$$




$$
\begin{aligned}
\tau_{5}= & m_{5} \widetilde{\dot{v}}_{c 5}{ }^{T} \cdot v_{c 5, \dot{\theta} 5}+m_{6} \tilde{\dot{v}}_{c 6}^{T} \cdot v_{c 6, \dot{\theta} 5}+m_{7} \widetilde{\dot{v}}_{c 7}{ }^{T} \cdot v_{c 7, \dot{\theta} 5} \\
& +\widetilde{N}_{5} \widetilde{\omega}_{5, \dot{\theta} 5}+\widetilde{N}_{6} \widetilde{\omega}_{6, \dot{\theta} 5}+\widetilde{N}_{7} \widetilde{\omega}_{7, \dot{\theta} 5}, \\
\tau_{6}= & m_{6} \widetilde{\dot{v}}_{c 6}{ }^{T} \cdot v_{c 6, \dot{\theta} 6}+m_{7} \widetilde{\dot{v}}_{c 7}^{T} \cdot v_{c 7, \dot{\theta} 6}+\widetilde{N}_{6} \widetilde{\omega}_{6, \dot{\theta} 6} \\
& +\widetilde{N}_{7} \widetilde{\omega}_{7, \dot{\theta} 6}, \\
\tau_{7}= & m_{7} \widetilde{\dot{v}}_{c 7}{ }^{T} \cdot v_{c 7, \dot{\theta} 7}+\widetilde{N}_{7} \widetilde{\omega}_{7, \dot{\theta} 7} .
\end{aligned}
$$

According to the different computing requirements, (20) is rewritten in a recursive way in (21).

In this 7-DOF robot, $n=7$ and $i=j=1 \sim 7$.

$$
\begin{aligned}
\tau_{j}= & \sum_{i=j}^{n} m_{i} \widetilde{\dot{v}}_{c i}{ }^{T} \cdot v_{c i, \dot{\theta} j} \\
& +\sum_{i=j}^{n}\left(I_{n} \widetilde{\dot{\omega}_{n}}+\widetilde{\omega_{n}} \times\left(I_{n} \cdot \widetilde{\omega_{n}}\right)\right)^{T} \widetilde{\omega}_{i, \dot{\theta} j} .
\end{aligned}
$$

Based on the established dynamic model for 7-DOF modular robot, the conclusion of the mission planning is put in (20). The main parameters required for the dynamic model are shown in Table 3. The coordinates of mission planning changes are shown in Figure 5 for end effector.

The results of the mission in the established model are shown in Figure 6 . And the torque parameters in Figure 6 are the same after Adams.

\section{Establish the Solid Dynamical Model and Simulation}

Based on the joint design of parameters and input conditions, the solid dynamical model is established by Proe and Adams for 7-DOF modular robot. The solid dynamical model is shown in Figure 7. In order to get closer to the real situation of the assembly and working conditions, the parts of part characteristic are combined in the process of modeling. The solid dynamical model is subjected to gravity in its natural state. In the working state by Coriolis force, the calculation results are automatically incorporated into the calculation of the solid model.

Substituting the results of the mission planning into (1) to (3), it gives the amount of displacement and velocity change per unit time. It means that from the algebraic formula we obtain the discrete point numerical solution. Considering the rate of change as a conditional input to the solid model, the task can be dynamic changes in the implementation of the situation.
After that, the conclusion of the mission planning in Figure 5 (the discrete point numerical solution) is put into the solid dynamical model in Figure 7. And the results of torque response are shown in Figure 8 from Joint 1 to Joint 7 . It can be seen from Figure 8 that the maximum torque change rate is joint 2 during the mission time, and the torque variation of Joint 1 has positive and negative fluctuation.

\section{Establish the Improved Kane Model}

There is an error between each model and the true value. The error of the Kane model is $\varepsilon$ from (21) to (27). And $\varepsilon$ is expressed in

$$
\varepsilon=T-T_{\text {theory }}
$$

The parameter $T$ is the torque in the solid dynamical model. The parameter $T_{\text {theory }}$ is the torque in the established Kane dynamical model and is equivalent to $\tau_{j}$.

When the model error can be improved, the improved torque $T^{\prime}$ exists and the improved error $\varepsilon^{\prime}$ is expressed in

$$
\varepsilon^{\prime}=T-\left(T_{\text {theory }}+T^{\prime}\right) .
$$

From Formulas (22) and (23), $T^{\prime}$ can be solved and is shown in

$$
T^{\prime}=T-T_{\text {theory }}-\varepsilon^{\prime}=\varepsilon-\varepsilon^{\prime}
$$

The improved error is far less than the error of the Kane model. It means $\varepsilon^{\prime} \ll \varepsilon$. So the improved torque is approximately equal to the error of the Kane model, and $T^{\prime}$ is expressed in

$$
T^{\prime} \approx \varepsilon
$$

The improved torque $T^{\prime}$ is related to the time $t$. And the time $t$ is the order of function $x$ about the improved torque $T^{\prime}$. We can get the improved torque $T^{\prime}{ }_{i}(t)$ which is expressed as follows:

$$
T_{i}^{\prime}(t)=\sum_{j=0}^{x} C_{i j} \cdot t^{j} .
$$

$C_{i j}$ is the undetermined coefficient matrix, $t$ is the execution time in the mission, $i$ is the function order, $j$ is number of the joint, and $x$ is function variable order. 
TABLE 3: The parameters of dynamic model.

\begin{tabular}{|c|c|c|c|c|c|c|c|c|}
\hline Designation & Unit & Joint 1 & Joint 2 & Joint 3 & Joint 4 & Joint 5 & Joint 6 & Joint 7 \\
\hline Joint quality & $\mathrm{kg}$ & 13.6 & 13.6 & 9.79 & 5.95 & 4.64 & 3.33 & 1.52 \\
\hline \multicolumn{9}{|l|}{ Mass center } \\
\hline$x$ & $\mathrm{~mm}$ & 0 & 1 & 0 & 85 & 0 & 0 & 0 \\
\hline$y$ & $\mathrm{~mm}$ & 0 & 100 & 0 & 0 & 58.43 & 0 & 0 \\
\hline$z$ & $\mathrm{~mm}$ & -100 & 0 & -129.53 & 111 & 0 & -75 & 0 \\
\hline Inertia tensor & $\mathrm{kg} \cdot \mathrm{mm}^{2}$ & Formula (7a) & Formula (7b) & Formula $(7 c)$ & Formula (7d) & Formula (7e) & Formula (7f) & Formula $(7 \mathrm{~g})$ \\
\hline \multicolumn{9}{|c|}{ Generalized displacement } \\
\hline$x$ & $\mathrm{~mm}$ & 0 & 0 & 0 & 0 & 170 & 0 & 0 \\
\hline$y$ & $\mathrm{~mm}$ & 0 & 0 & 0 & 0 & 0 & 0 & 0 \\
\hline$z$ & $\mathrm{~mm}$ & 420 & 147 & 535 & 0 & 0 & 401 & 91 \\
\hline Initial conditions & \multicolumn{8}{|c|}{$\omega_{0}=0, \dot{\omega}_{0}=0, v_{0}=0, \dot{v}_{0}=\left[\begin{array}{lll}0 & 0 & g\end{array}\right]^{T}$} \\
\hline
\end{tabular}
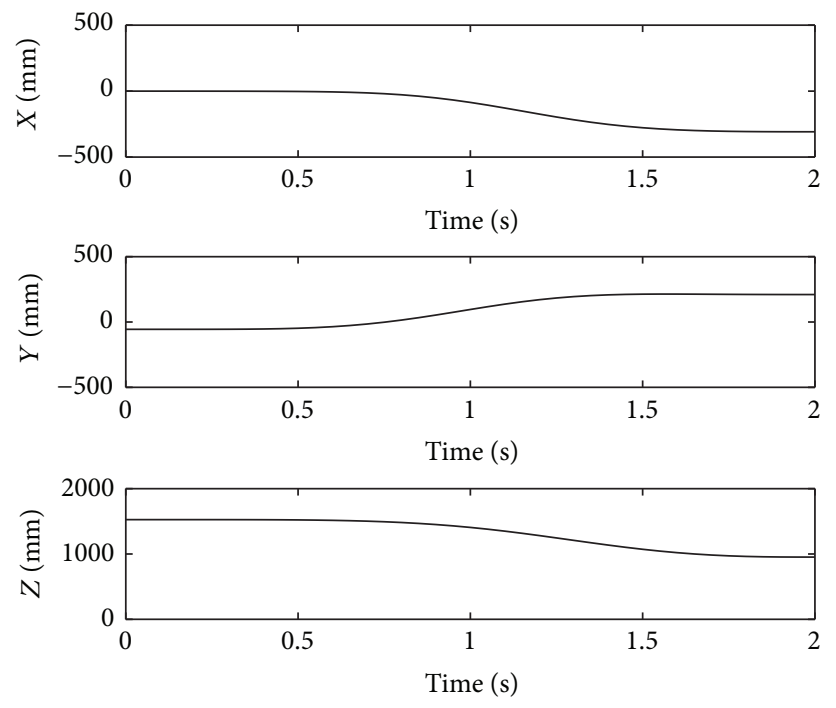

FIGURE 5: End joint coordinate change curves.

\section{The Experimental Data Analysis and Improved Function Solving}

The robot is reconfigurable by the designed joint. Figure 9 shows the reconstruction from 1-DOF to 7-DOF. The robot can work alone in each degree of freedom, or in a degree of freedom in the different configuration transformation. Considering communication using the Can-open, so the different configurations of the reconfiguration are flexible and convenient.

According to the reconfigurable robot, we are able to build and simulate the dynamics of the mathematical and solid models. From the experimental data obtained before, we can draw the following analysis results.

The torque response is different in each Joint from Joint 1 to Joint 7. The torque variations are obtained that the result of the solid dynamical model in Figure 8 subtracts the result of the Kane dynamical model in Figure 6. And the torque variation curves are shown in Figures 10-16 of each joint.
The model error is determined that the result of solid dynamical model of each joint subtracts the result of the Kane dynamical model of each joint from Figure 10 to Figure 16. After that, the error curves in the mission each joint are shown in Figure 17.

The maximum error variance from Joint 2 and Joint 3 can be seen; Joint 1 also showed positive and negative fluctuations. The difference of Joint 6 and Joint 7 is small, or even a partial coincidence. And this is related to its smaller execution torque. In addition, it can be seen that, with the task of time, the overall error was small to large changes.

Considering analytical dynamic model and the error distribution in Figure 17, we can correct the joint error that finds the error of the law of the joints and algebra.

The experimental data are first fitting and linearization from Figure 17, and then the distribution is analyzed. The part of fitting data is shown in Table 4.

According to Table 4, the function order is five that is determined by data fitting. And the undetermined coefficient matrix is solved and expressed in 
TABLE 4: The part of experimental data fitting. Unit: $\mathrm{kg} \cdot \mathrm{mm}^{2} / \mathrm{s}^{2}$.

\begin{tabular}{lccccccc}
\hline Number & Joint 1 & Joint 2 & Joint 3 & Joint 4 & Joint 5 & Joint 6 & Joint 7 \\
\hline 1 & $-1.8 E+03$ & $1.8 E+04$ & $2.4 E+03$ & $-1.8 E+03$ & $2.8 E+02$ & $3.1 E+01$ & $2.1 E+01$ \\
2 & $-6.2 E+05$ & $2.5 E+06$ & $-2.8 E+06$ & $-5.9 E+05$ & $2.3 E+04$ & $3.8 E+06$ & $9.2 E+03$ \\
3 & $-1.6 E+05$ & $4.2 E+05$ & $6.0 E+06$ & $-1.5 E+06$ & $8.2 E+05$ & $3.9 E+06$ & $1.7 E+04$ \\
4 & $2.6 E+06$ & $-4.1 E+06$ & $2.2 E+07$ & $-3.0 E+06$ & $1.6 E+06$ & $3.6 E+06$ & $-5.6 E+03$ \\
5 & $9.1 E+06$ & $-9.1 E+06$ & $3.8 E+07$ & $-6.0 E+06$ & $2.4 E+06$ & $2.8 E+06$ & $-4.3 E+04$ \\
6 & $1.6 E+07$ & $-2.2 E+07$ & $4.4 E+07$ & $-1.1 E+07$ & $1.7 E+06$ & $2.1 E+06$ & $7.2 E+03$ \\
7 & $9.4 E+05$ & $-8.1 E+07$ & $4.8 E+07$ & $-2.1 E+07$ & $-3.3 E+06$ & $4.2 E+05$ & $1.3 E+05$ \\
8 & $-9.4 E+06$ & $-9.9 E+07$ & $5.5 E+07$ & $-2.4 E+07$ & $-5.0 E+06$ & $-3.7 E+05$ & $7.1 E+04$ \\
9 & $-1.2 E+07$ & $-1.0 E+08$ & $6.2 E+07$ & $-2.4 E+07$ & $-6.0 E+06$ & $-6.4 E+05$ & $1.9 E+04$ \\
10 & $-5.6 E+06$ & $-9.1 E+07$ & $7.2 E+07$ & $-2.2 E+07$ & $-6.7 E+06$ & $-5.9 E+05$ & $1.1 E+03$ \\
\hline
\end{tabular}

TABLE 5: The increase of each joint dynamics model.

\begin{tabular}{lccccccc}
\hline Number & Joint 1 & Joint 2 & Joint 3 & Joint 4 & Joint 5 & Joint 6 & Joint 7 \\
\hline Percent (\%) & 33.28 & 32.87 & 34.14 & 33.15 & 34.14 & 34.15 & 33.30 \\
\hline
\end{tabular}

$$
C_{i j}=\left[\begin{array}{cccccc}
1.4 E+06 & -2.4 E+07 & 2.4 E+07 & 1.0 E+08 & -1.3 E+08 & 3.7 E+07 \\
4.0 E+06 & -5.7 E+07 & 2.3 E+08 & -3.2 E+08 & 1.3 E+08 & -1.1 E+07 \\
3.7 E+05 & -1.1 E+08 & 5.1 E+08 & -6.0 E+08 & 2.9 E+08 & -4.9 E+07 \\
3.4 E+05 & -1.2 E+07 & 4.5 E+07 & -8.2 E+07 & 4.4 E+07 & -7.4 E+06 \\
6.1 E+04 & -1.1 E+07 & 5.7 E+07 & -7.4 E+07 & 3.4 E+07 & -5.4 E+06 \\
1.3 E+06 & 2.2 E+07 & -5.7 E+07 & 5.8 E+07 & -2.8 E+07 & 5.3 E+06 \\
-3.8 E+04 & 9.5 E+05 & -3.9 E+06 & 5.5 E+06 & -3.1 E+06 & 5.9 E+05
\end{array}\right]
$$

The torque correction value of the correction function is divided by the original Kane model value. And when the accuracy of the original Kane is 35\%, the increase of the joint dynamics is model shown in Table 5 . The average increase rate of the dynamic model accuracy is $33.57 \%$.

Therefore, the improved torque of joint number $j$ is expressed as follows:

$$
T_{i}^{\prime}(t)=\sum_{i=0}^{5} c_{i j} \cdot t^{j}
$$

The improved function is determined for 7-DOF modular robot, as expressed in

$$
\begin{aligned}
T^{\prime}(t) & =\left[\begin{array}{lllll}
T_{1}^{\prime}(t) & T_{2}^{\prime}(t) & \cdots & T_{i}^{\prime}(t)
\end{array}\right]^{T} \\
& =C_{i j} \cdot\left[\begin{array}{llll}
t^{0} & t^{1} & \cdots & t^{j}
\end{array}\right]^{T} .
\end{aligned}
$$

The improved function $T^{\prime}$ is 5-order constant coefficient function. And the improved Kane Dynamic Model is expressed as follows:

$$
\begin{aligned}
T_{n}= & \widetilde{N}_{n}+T^{\prime}(t) \\
= & \left(I_{n} \widetilde{\dot{\omega}_{n}}+\widetilde{\omega_{n}} \times\left(I_{n} \cdot \widetilde{\omega_{n}}\right)\right)^{T} \\
& +\left[\begin{array}{llll}
T_{1}^{\prime}(t) & T^{\prime}{ }_{2}(t) & \cdots & T_{i}^{\prime}(t)
\end{array}\right]^{T} .
\end{aligned}
$$

Formula (30) is equivalent to Formula (37), as shown in

$$
\tau_{\theta}=\tau_{\theta_{j}}+T^{\prime}(t)
$$




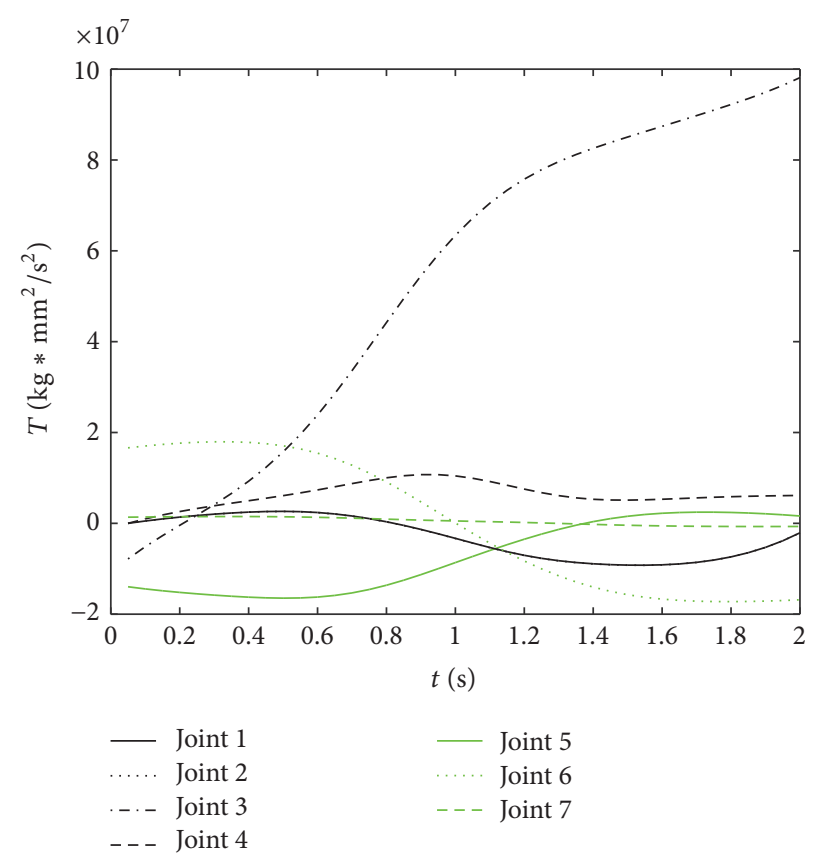

Figure 6: Mission execution results of the Kane dynamic model.

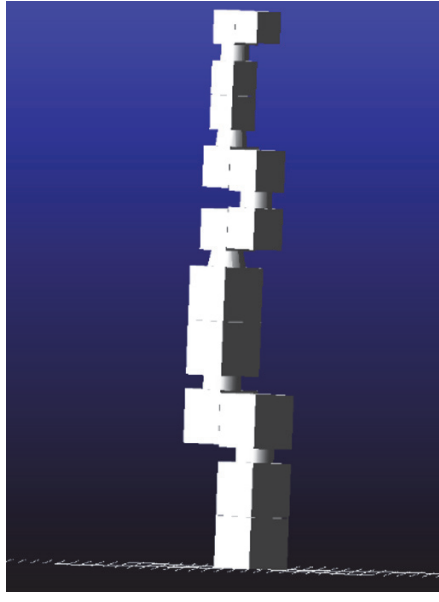

FIgURE 7: The solid dynamical model of 7-DOF modular robot.

\section{Conclusion}

We proposed an improved Kane dynamic model theory for 7 -DOF modular robot in this paper. The Kane dynamic model and the solid dynamic model are established, respectively, for the robot. After that, the experimental results are obtained from the simulation experiment of typical task in the established dynamic models. By the analysis model of error, the equation of the improved torque $T^{\prime}{ }_{i}(t)$ is derived and proposed. The improved Kane dynamic model is established for the modular robot that uses $T^{\prime}{ }_{i}(t)$. Based

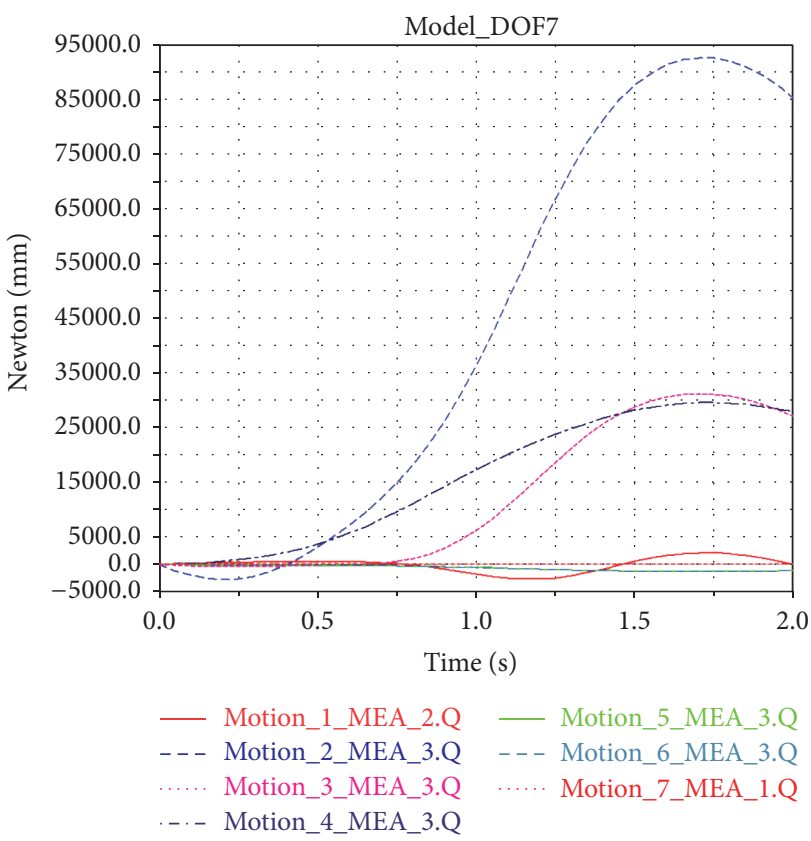

FIGURE 8: The torque response curves for the solid dynamical model.

on the experimental data, the undetermined coefficient matrix $C_{i j}$ is five-order linear that is proved in 7-DOF modular robot. And the explicit formulations are solved of the Kane dynamic model and can be used in control system. 


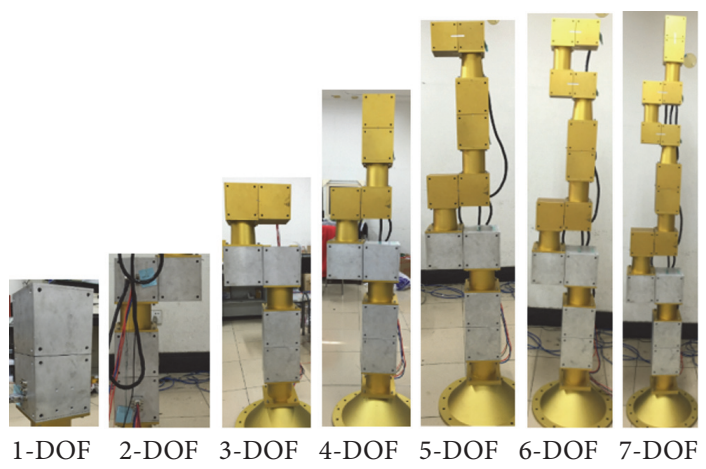

FIgURE 9: The Joint 1 torque response.

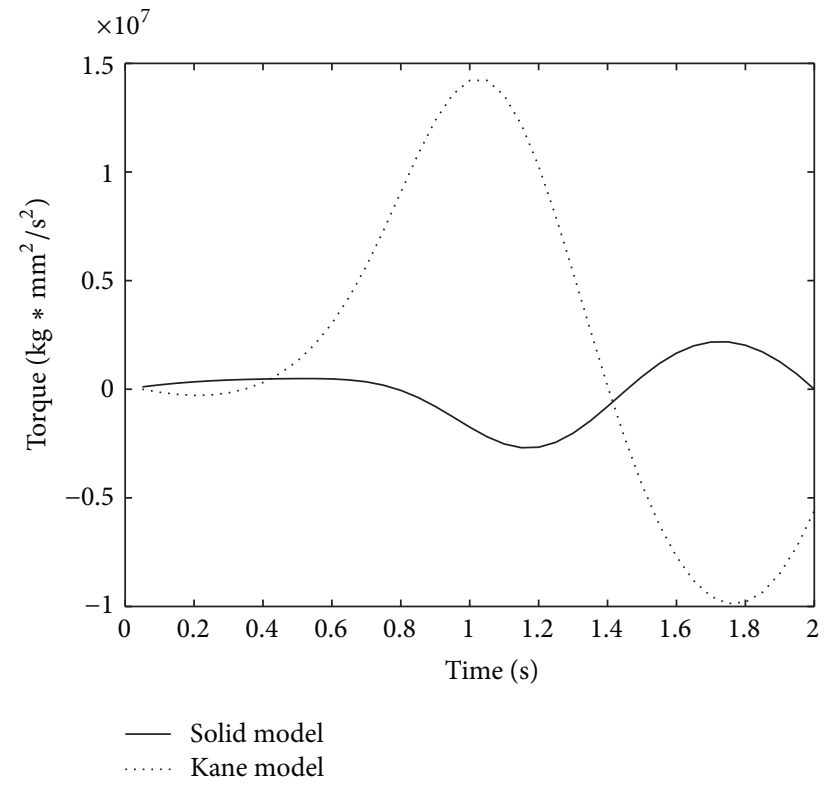

FIgURE 10: The Joint 1 torque response.

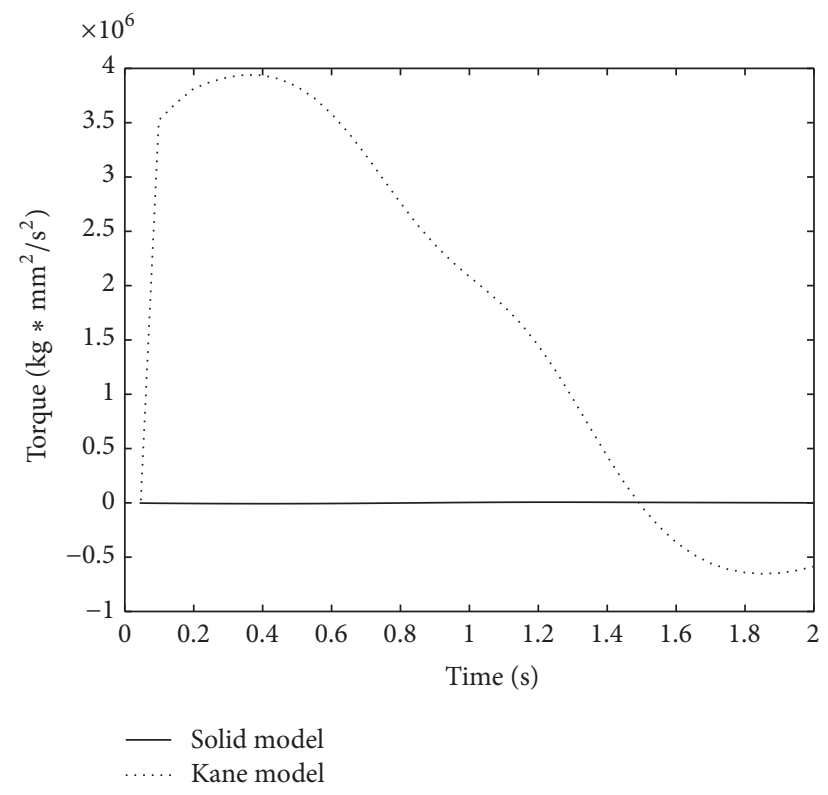

Figure 11: The Joint 2 torque response.

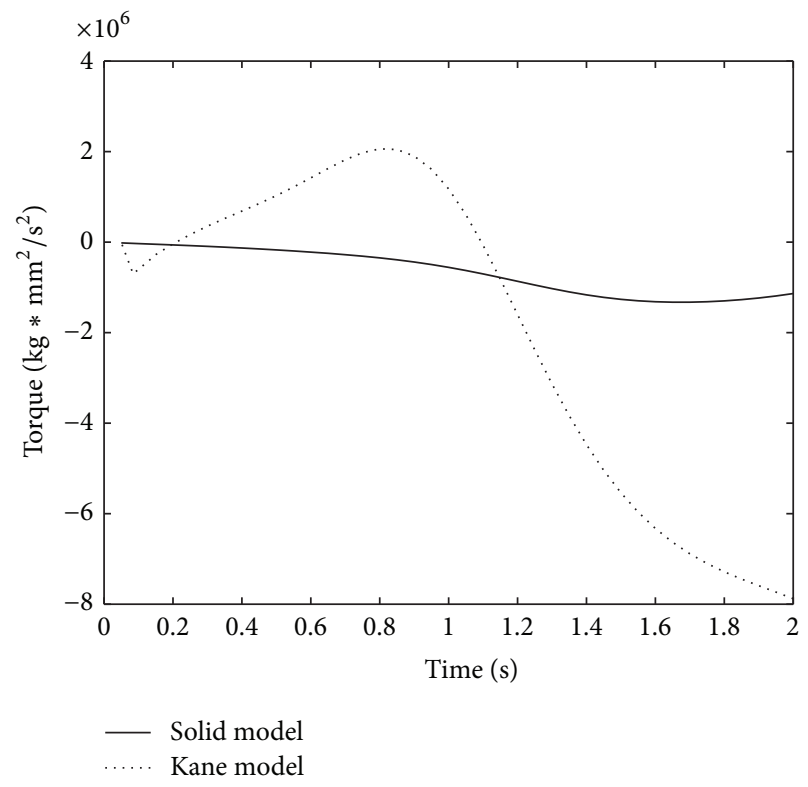

FIGURE 12: The Joint 1 torque response.

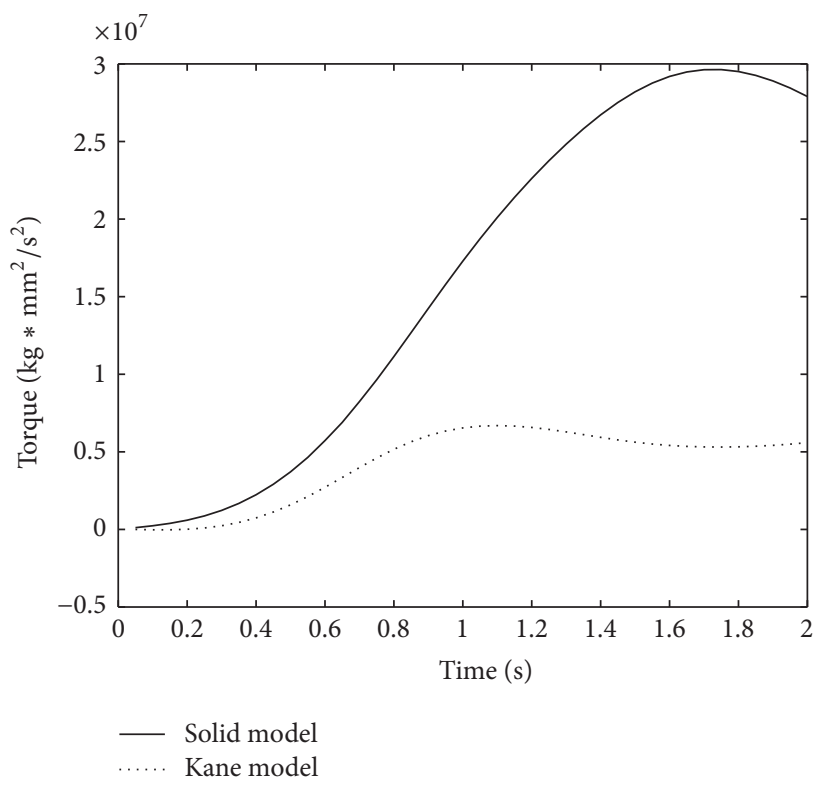

FIGURE 13: The Joint 2 torque response. 


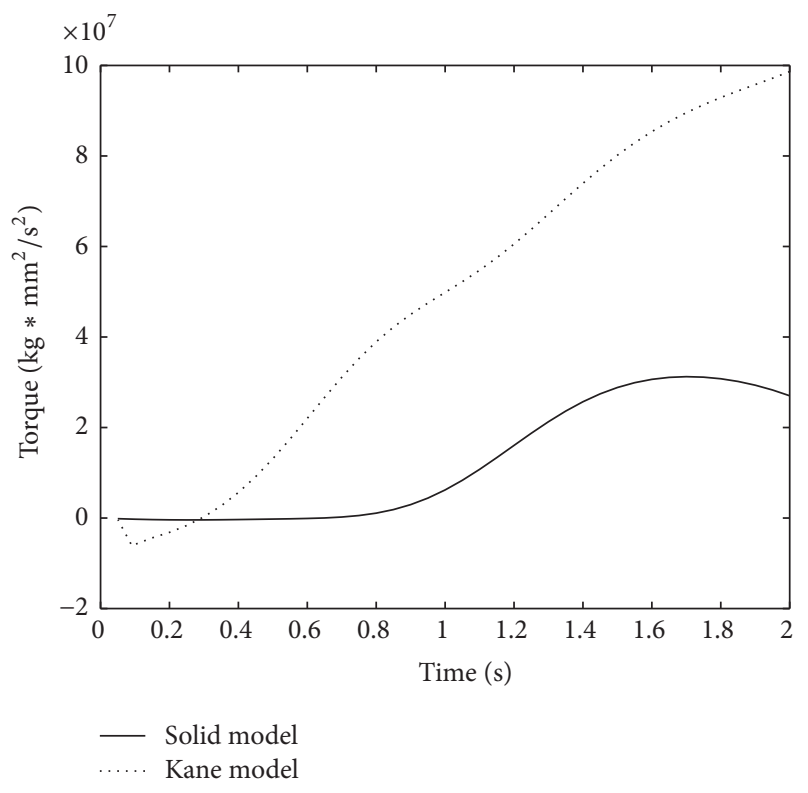

Figure 14: The Joint 1 torque response.

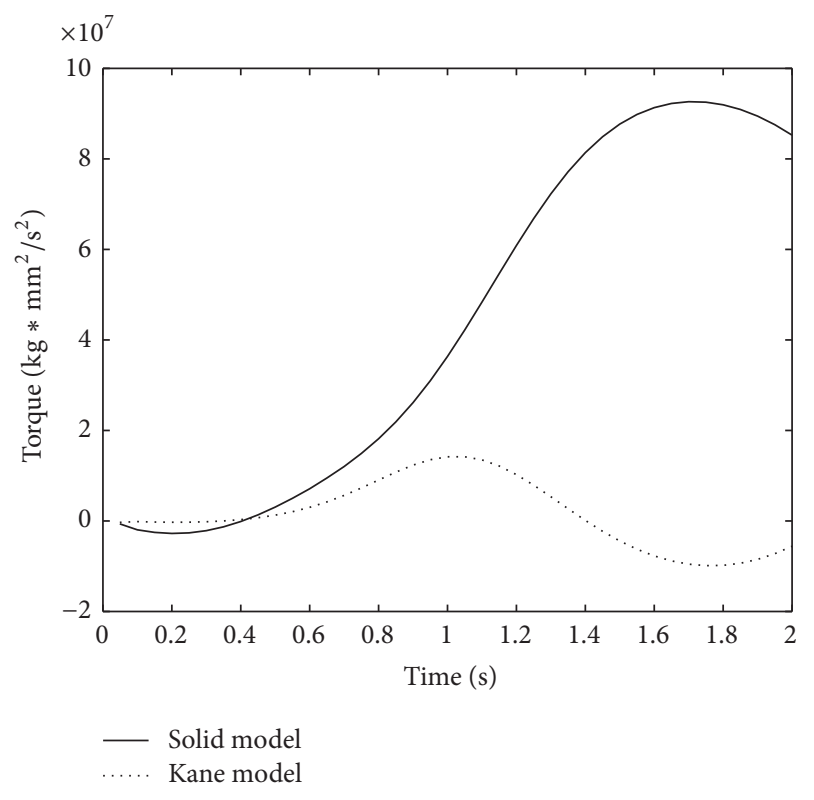

FIGURE 15: The Joint 2 torque response.

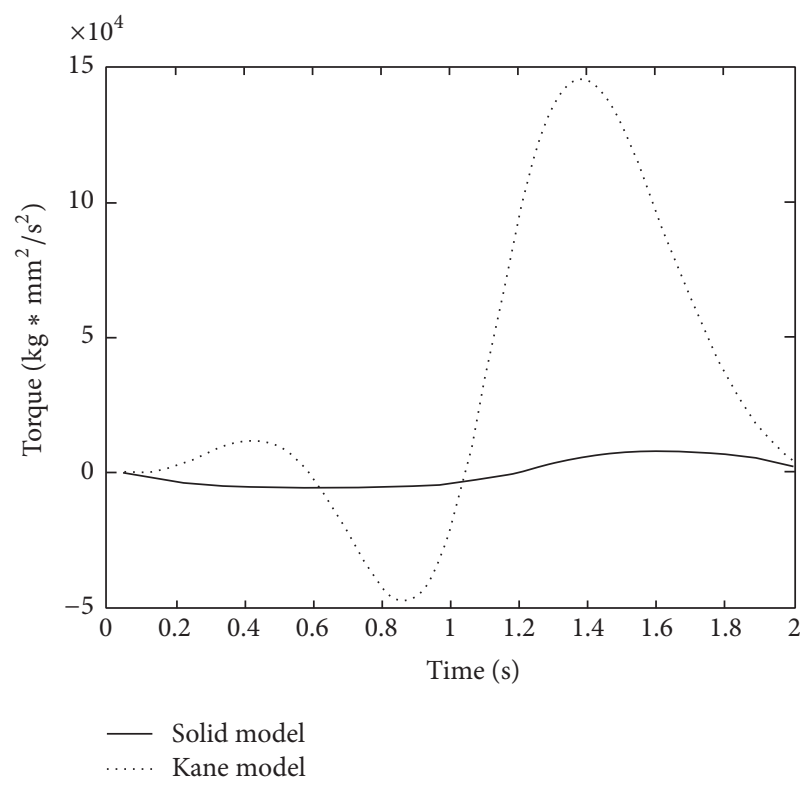

FIGURE 16: The Joint 1 torque response.

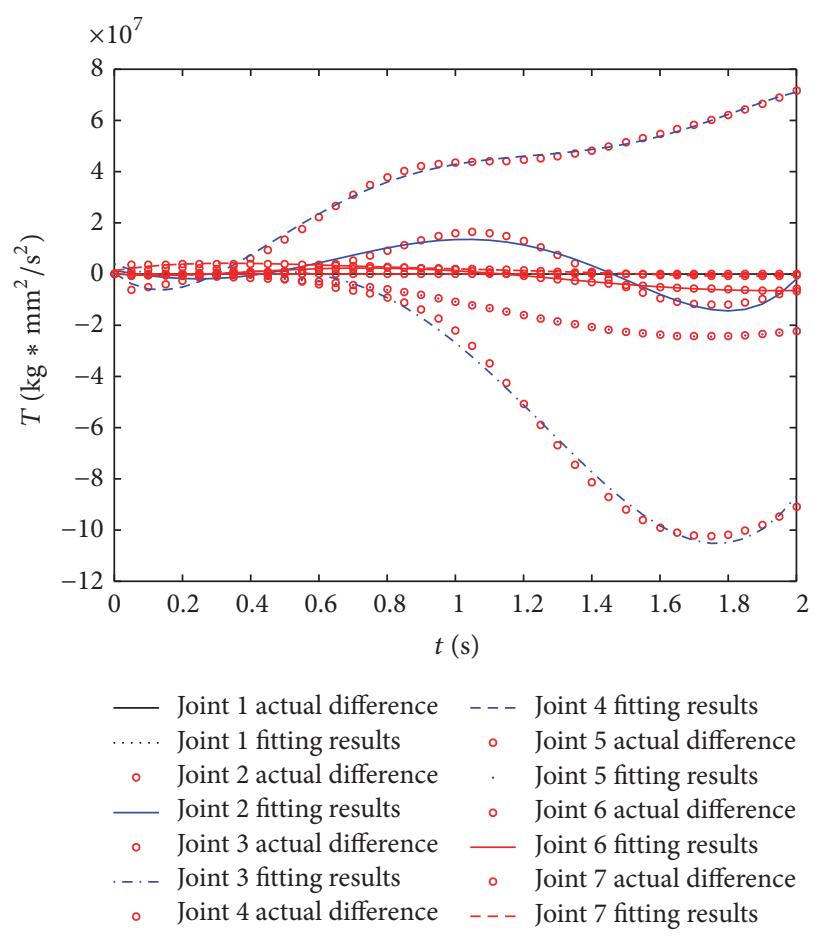

FIGURE 17: The curves of the model error of each joint.

\section{Competing Interests}

The authors declare that there is no conflict of interests regarding the publication of this paper.

\section{Acknowledgments}

This research was supported by the National Basic Research Program of China (no. 2013CB733000).

\section{References}

[1] S. Funiak, P. Pillai, M. P. Ashley-Rollman, J. D. Campbell, and S. C. Goldstein, "Distributed localization of modular robot ensembles," International Journal of Robotics Research, vol. 28, no. 8, pp. 946-961, 2009.

[2] J. Deng, P. Wang, Q. Meng, H. Shen, J. Chen, and M. Luo, "Design and development on a reconfigurable modular joint of the robot arm," in Advances in Reconfigurable Mechanisms and 
Robots II, vol. 36 of Mechanisms and Machine Science, pp. 705714, Springer, Berlin, Germany, 2016.

[3] W. Weizhong, Key Technologies for Reconfigurable Modular Robot System, Harbin Institute of Technology, 2007.

[4] R. A. Iureva, E. O. Raskin, I. I. Komarov, N. K. Maltseva, and M. E. Fedosovsky, Industrial Robot's Vision Systems, SPIE OPT, OInternational Society for Optics and Photonics, 2016.

[5] Y. Li, H. Sun, Y. Zhang, M. Chu, Q. Jia, and X. Lan, "Characteristic analysis and fluctuation control for a underactuated spherical underwater robot," Journal of Vibroengineering, vol. 16, no. 1, pp. 42-56, 2014.

[6] Y. Li, H. Sun, M. Chu, Y. Zhang, Q. Jia, and X. Lan, "Experiment, simulation and analysis on coupling hydrodynamic forces under key parameters for a spherical underwater exploration robot," Journal of Vibroengineering, vol. 16, no. 6, pp. 3014-3025, 2014.

[7] H. Sun, K. Hou, and Q. Jia, "Development, analysis and control of a spherical aerial vehicle," Journal of Vibroengineering, vol. 15, no. 2, pp. 1069-1080, 2013.

[8] Y. Wang, X. Gao, H. Sun, Q. Jia, W. Zhao, and M. Du, "Research on control method based on real-time operational reliability evaluation for space manipulator," Advances in Mechanical Engineering, vol. 6, no. 8, Article ID 179293, 2014.

[9] A. Schweikard and F. Ernst, "Robot kinematics," in Medical Robotics, pp. 63-122, Springer, Berlin, Germany, 2015.

[10] C. Ayiz and S. Kucuk, "The kinematics of industrial robot manipulators based on the exponential rotational matrices," in Proceedings of the IEEE International Symposium on Industrial Electronics (ISIE '09), pp. 977-982, IEEE, Seoul, Republic of Korea, July 2009.

[11] J. R. J. De, P. C. J. Humberto, Z. Zamudio, and A. J. Salinas, "Comparison of two quadrotor dynamic models," IEEE Latin America Transactions, vol. 12, no. 4, pp. 531-537, 2014.

[12] Y. Zhao, Z. Duan, and G. Wen, "Distributed finite-time tracking of multiple Euler-Lagrange dynamics without velocity measurements," in Proceedings of the Control Conference, pp. 1688-1703, August 2013.

[13] X. Ge and J. Jin, "Dynamics analyze of a dual-arm space robot system based on Kane's method," in Proceedings of the 2nd International Conference on Industrial Mechatronics and Automation (ICIMA '10), pp. 646-649, IEEE, Wuhan, China, May 2010.

[14] K. Yang, X. Wang, T. Ge, and C. Wu, "A dynamic model of ROV with a robotic manipulator using Kane's method," in Proceedings of the 5th International Conference on Measuring Technology and Mechatronics Automation, pp. 9-12, IEEE Computer Society, 2013.

[15] Y. Li and Q. Xu, "Kinematic analysis and dynamic control of a 3PUU parallel manipulator for cardiopulmonary resuscitation," in Proceedings of the 12th International Conference on Advanced Robotics (ICAR '05), pp. 344-351, IEEE, Seattle, Wash, USA, July 2005.

[16] Z. Xuchong, C. Zhong, and Z. Xianmin, "Kinematic analysis and optimal design of a 3D compliant probe," in Proceedings of the IEEE International Conference on Robotics and Biomimetics (ROBIO '12), pp. 1176-1181, IEEE, Guangzhou, China, December 2012.

[17] C. Yang, H. Ma, and M. Fu, "Robot kinematics and dynamics modeling," in Advanced Technologies in Modern Robotic Applications, 2016.
[18] R. Grepl, P. Čoupek, J. Radoš, J. Konvičný, and M. Krejčiřík, "Modeling of kinematics, dynamics and design of electronics control unit for an experimental robot with hybrid locomotion," in Advanced Mechatronics Solutions, Springer, Berlin, Germany, 2016.

[19] O. Rios, "Method of influence coefficients for kinematic and dynamic modeling of robotic systems," IEEE Transactions on Robotics, vol. 32, no. 1, pp. 236-245, 2015.

[20] H. Shen, H. Wu, and B. Chen, "High effective inverse dynamics modelling for multi-DOF serial robots," China Mechanical Engineering, no. 1, pp. 20-24, 2016.

[21] Y. Xu, Y.-H. Zhang, W.-H. Liu, X.-G. Duan, and L. Gao, "Dynamics modeling and smooth control of flexible robot joint," in Proceedings of the 12th IEEE International Conference on Mechatronics and Automation (ICMA '15), pp. 631-636, Beijing, China, August 2015.

[22] S. R. Ahmadzadeh, Modal approach to modeling of hyperredundant robot manipulators dynamics and design of fuzzy controller for the system [M.S. thesis], Bahonar University, Kerman, Iran, 2004.

[23] Y. Yun and Y. Li, "Design and analysis of a novel 6-DOF redundant actuated parallel robot with compliant hinges for high precision positioning," Nonlinear Dynamics, vol. 61, no. 4, pp. 829-845, 2010.

[24] Y. Yun and Y. Li, "A general dynamics and control model of a class of multi-DOF manipulators for active vibration control," Mechanism \& Machine Theory, vol. 46, no. 10, pp. 1549-1574, 2011.

[25] Y. Yun and Y. Li, "A general model of a kind of parallel manipulator for active control based on KANE's dynamics," in Proceedings of the IEEE Asia Pacific Conference on Circuits and Systems (APCCAS '08), pp. 1830-1833, IEEE, Macao, China, December 2008. 


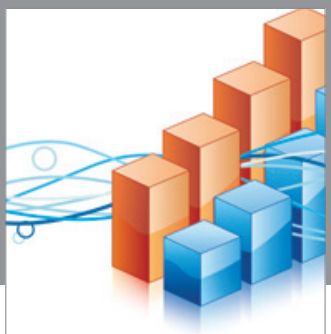

Advances in

Operations Research

vatem alat4

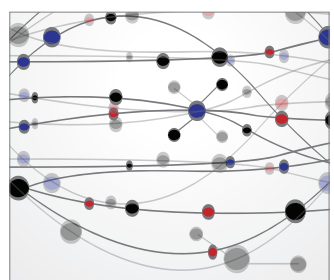

\section{The Scientific} World Journal
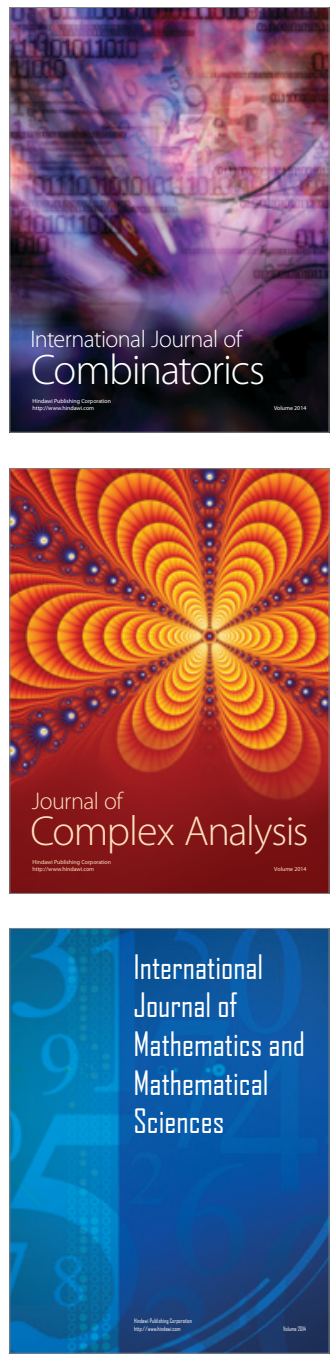
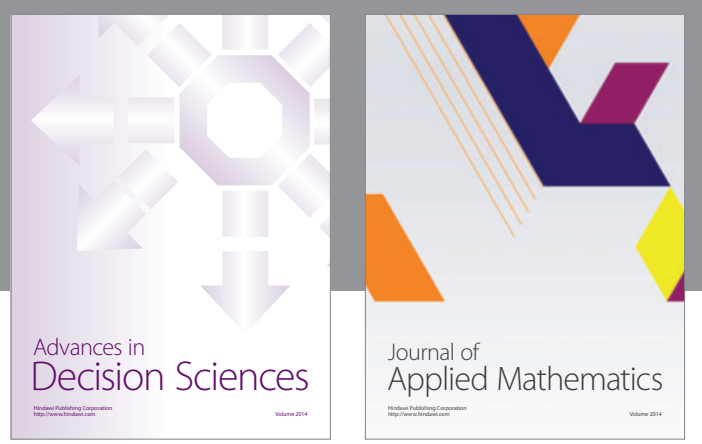

Algebra

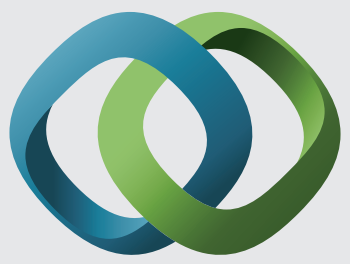

\section{Hindawi}

Submit your manuscripts at

https://www.hindawi.com
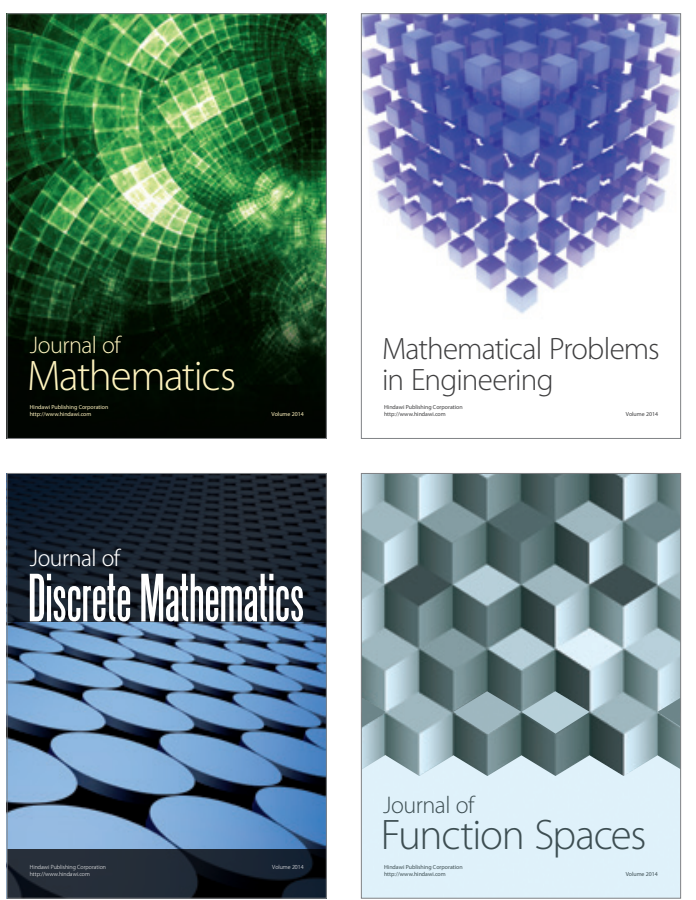

Mathematical Problems in Engineering
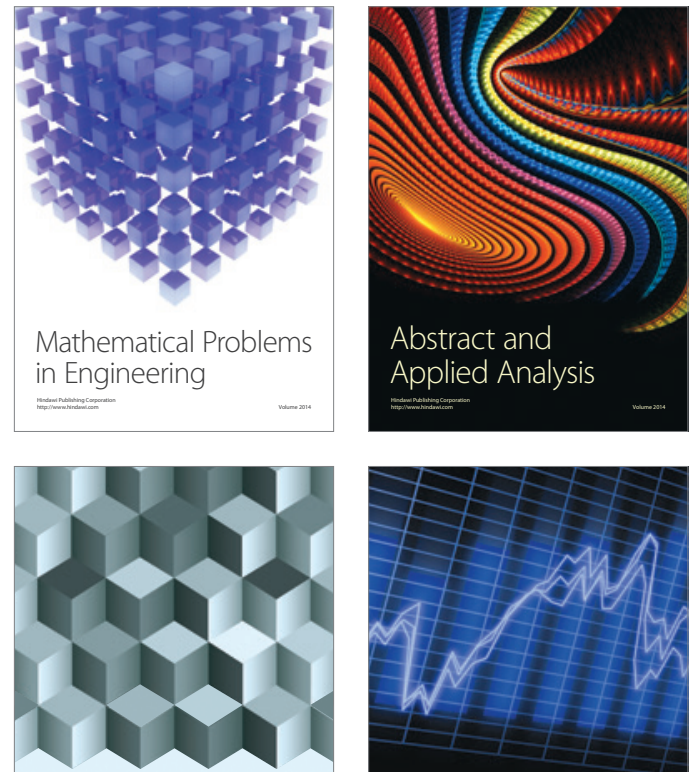

Journal of

Function Spaces

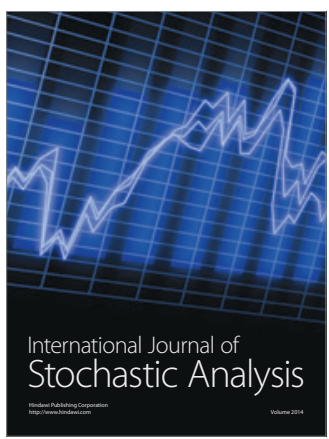

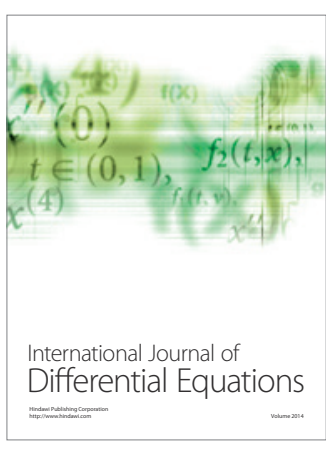
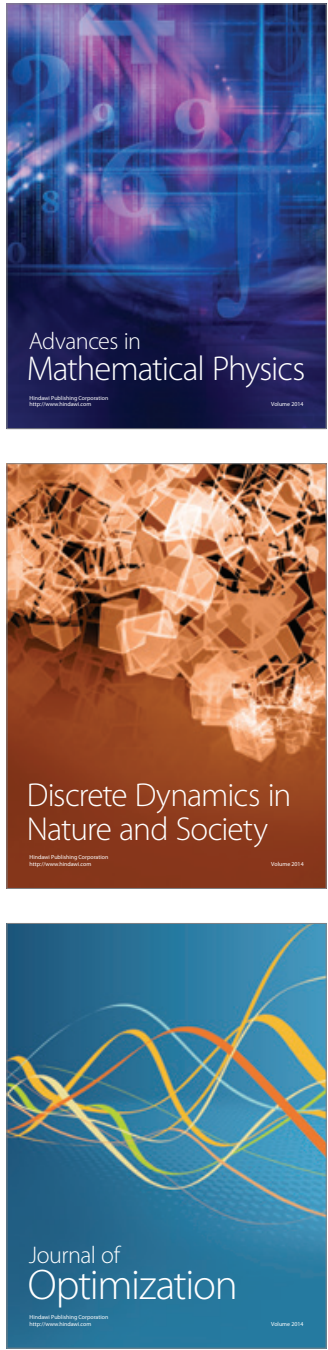